\title{
The Thomas-Fermi-Theory as Result of a Strong-Coupling-Limit
}

\author{
Bernhard Baumgartner \\ Institut für theoretische Physik der Universität Wien, A-1090 Wien, Austria
}

\begin{abstract}
The energy of non-relativistic neutral atoms is shown to approach asymptotically the Thomas-Fermi-energy, when the charge of the nucleus tends to infinity.
\end{abstract}

\section{Introduction}

As is well known, the Thomas-Fermi- (T.F.-) theory for atoms results by treating the electrons as a locally ideal Fermi-gas in the mean field created by the nucleus and the other electrons. We show that this scheme becomes correct if we take the limit of infinitely large atoms in nonrelativistic quantummechanics. (This result has also been announced by Lieb and Simon [1].) First we show that the groundstate-energy in a mean-field-theory, which supplies us with lower bounds to the energy of atoms, tends to the T.F.-energy. In the second step the density in ground states of related Hamiltonians is shown to converge in a certain sense to the T.F.density. Finally we take the expectation value of the nonrelativistic Hamiltonian for atoms in the above mentioned ground state and show that it also gives asymptotically the T.F.-energy.

\section{The Limit of a Lower Bound}

We use a Hamiltonian, which gives lower bounds to the energies of atoms, recently found by P. Hertel et al. [2]:

$$
\begin{aligned}
h_{N}= & \sum_{i=1}^{N}\left(P_{i}^{2} / 2 m-N\left|x_{i}\right|^{-1}+\phi_{N}\left(x_{i}\right)\right. \\
& -\frac{1}{2}\left\{\int \phi_{N}(x) n_{N}(x) d^{3} x+3 N\left(\pi / N \int n_{N}(x)^{2} d^{3} x\right)^{1 / 3}\right\}, \\
\phi_{N}(x)= & \int|x-y|^{-1} n_{N}(y) d^{3} y,
\end{aligned}
$$


$n_{N}(x)$ is an arbitrary density in $L^{2}\left(R^{3}\right)$

$$
h_{N} \leqq H_{N}=\sum_{i=1}^{N}\left(P_{i}^{2} / 2 m-N\left|x_{i}\right|^{-1}\right)+\sum_{i<j}\left|x_{i}-x_{j}\right|^{-1} .
$$

We mimic T.F.-theory by setting

$$
n_{N}(x)=N^{2} n\left(N^{1 / 3} x\right)
$$

which implies

$$
\phi_{N}(x)=N^{4 / 3} \phi\left(N^{1 / 3} x\right) \quad \text { with } \quad \phi(x)=\int|x-y|^{-1} n(y) d^{3} y
$$

and make the unitary scale-transformation

$$
N^{1 / 3} x \mapsto x, N^{-1 / 3} p \mapsto p,
$$

such that [with $\left.V(x)=\phi(x)-|x|^{-1}\right]$

$$
\begin{aligned}
h_{N} \cong \tilde{h}_{N}= & N^{2 / 3} \sum_{i=1}^{N} p_{i}^{2} / 2 m+N^{4 / 3} \sum_{i=1}^{N} V\left(x_{i}\right)-N^{7 / 3} \frac{1}{2} \int \phi(x) n(x) d^{3} x \\
& -N^{5 / 3} \frac{3}{2}\left(\pi \int n(x)^{2} d^{3} x\right)^{1 / 3} .
\end{aligned}
$$

According to the degeneracy caused by the spins we have to evaluate twice the sum of the $N / 2$ lowest eigenvalues of $N^{2 / 3} \frac{p^{2}}{2 m}-N^{4 / 3}\left(|x|^{-1}-\phi(x)\right)$. The limit $N \rightarrow \infty$ may be regarded either as the strong-coupling- or as the classical limit. The result is given by a theorem which is proven in [3]:

$$
\begin{aligned}
\mathscr{E}(n, V): & =\lim _{N \rightarrow \infty} N^{-7 / 3} \inf _{\psi,\|\psi\|=1}\left\langle\psi\left|h_{N}\right| \psi\right\rangle \\
& =(3 / 10 m)\left(3 \pi^{2}\right)^{2 / 3} \int \varrho(x, \mu)^{5 / 3} d^{3} x+\int V(x) \varrho(x, \mu) d^{3} x-\frac{1}{2} \int \phi(x) n(x) d^{3} x .
\end{aligned}
$$

The density is

$$
\varrho(x, \mu)=(\mu-V(x))^{3 / 2} \theta(\mu-V(x))
$$

where $\mu$ has to be chosen either such that $\int \varrho(x, \mu) d^{3} x=1$, or equal to zero if $\int \varrho(x, 0) d^{3} x=\|\varrho(x, 0)\|_{1}<1$. If we use for $n(x)$ the normalized $\left(\left\|\varrho_{\text {T.F. }}\right\|_{1}=1\right)$ T.F.density for atoms, $\varrho_{\text {T.F. }}(x)$, then also $\varrho(x, \mu)=\varrho_{\text {T.F. }}(x)$, and $E\left(\varrho_{\text {T.F. }}, \phi(x)-|x|^{-1}\right)$ equals the T.F.-energy $[1,4]$. Unfortunately, $\varrho_{\text {T.F. }}$ is not square-integrable (it has a singularity as $|x|^{-3 / 2}$ at $x \rightarrow 0$ ), but it may be approximated by squareintegrable $n_{v}(x)$ both in the $L^{5 / 3}$ and in the $L^{1}$-topology:

$$
\begin{aligned}
\exists\left\{n_{v}(x)\right\} \subset L^{2}\left(\mathbb{R}^{3}\right): & \lim _{v \rightarrow \infty}\left\|n_{v}-\varrho_{\text {T.F. }}\right\|_{1}=0 \\
& \lim _{v \rightarrow \infty}\left\|n_{v}-\varrho_{\text {T.F. }}\right\|_{5 / 3}=0 .
\end{aligned}
$$

The energy functional (8) is then continuous as $v \rightarrow \infty$ :

$$
\begin{aligned}
& \lim _{v \rightarrow \infty} \mathscr{E}\left(n_{v}, V_{v}\right)=E_{\text {T.F. }} \\
& =(3 / 10 m)\left(3 \pi^{2}\right)^{2 / 3} \int \varrho_{\text {T.F. }}(x)^{5 / 3} d^{3} x-\int|x|^{-1} \varrho_{\text {T.F. }}(x) d^{3} x \\
& \quad+\frac{1}{2} \int \varrho_{\text {T.F. }}(x)|x-y|^{-1} \varrho_{\text {T.F. }}(y) d^{3} x d^{3} y,
\end{aligned}
$$


and we have the desired asymptotic lower bound:

$$
\lim _{N \rightarrow \infty} \inf _{\psi,\|\psi\|=1}\left\langle\psi\left|H_{N}\right| \psi\right\rangle \geqq \lim _{N \rightarrow \infty} \inf _{\psi,\|\psi\|=1}\left\langle\psi\left|h_{N}\right| \psi\right\rangle=E_{\mathrm{T} . \mathrm{F} .} .
$$

\section{Convergence of Densities}

The asymptotic behavior of the ground-state energies $E_{N}(\alpha)$ of

$$
h_{N}(\alpha)=N^{-2 / 3} \sum_{i=1}^{N} p_{i}^{2} / 2 m+\sum_{i=1}^{N}\left(V\left(x_{i}\right)+\alpha w\left(x_{i}\right)\right)
$$

for spin- $\frac{1}{2}$-particles is as in (8), if $w$ is a test-function in $S\left(\mathbb{R}^{3}\right)^{1}$, namely:

$$
\begin{aligned}
& \lim _{N \rightarrow \infty} N^{-1} E_{N}(\alpha)=\mathscr{E}(\alpha) \\
& =(3 / 10 m)\left(3 \pi^{2}\right)^{2 / 3} \int \varrho(\alpha, x)^{5 / 3} d^{3} x+\int V(x) \varrho(\alpha, x) d^{3} x+\alpha \int w(x) \varrho(\alpha, x) d^{3} x . \\
& \varrho(\alpha, x)=\left(\mu_{\alpha}-V(x)-\alpha w(x)\right) \theta\left(\mu_{\alpha}-V(x)-\alpha w(x)\right) .
\end{aligned}
$$

Since the $E_{N}(\alpha)$ are concave functions of $\alpha$, there exist the right and left derivatives $E_{N^{+}}{ }^{\prime}(\alpha)$ and $E_{N^{\prime}}{ }^{\prime}(\alpha)$, which determine the bounds

$$
E_{N^{+}}{ }^{\prime}(0) \leqq N \int w(x) \varrho_{N}(x) d^{3} x \leqq E_{N^{-}}{ }^{\prime}(0)
$$

for

$$
\varrho_{N}(x)=\sum_{s_{1} \ldots s_{N}} \int\left|\psi_{N}\left(x, x_{2} \ldots x_{N} ; s_{1} \ldots s_{N}\right)\right|^{2} d^{3} x_{2} \ldots d^{3} x_{N}
$$

where $\psi_{N}$ is the ground-state-wavefunction of $h_{N}(0)$. We use Griffith's Lemma $[6,7]$ :

$$
\lim _{N \rightarrow \infty} N^{-1} E_{N^{+}}^{\prime}(0)=\lim _{N \rightarrow \infty} N^{-1} E_{N^{\prime}}^{\prime}(0)=d \mathscr{E}(\alpha) /\left.d \alpha\right|_{\alpha=0}
$$

which implies

$$
\lim _{N \rightarrow \infty} \int w(x) \varrho_{N}(x) d^{3} x=\int w(x) \varrho(0, x) d^{3} x .
$$

$\varrho(0, x)$ is the T.F.-density if we specify $V(x)$ in (13) to be $\int|x-y|^{-1} \varrho_{\text {T.F. }}(y) d^{3} y-$ $|x|^{-1}$.

\section{Asymptotic Behavior of an Upper Bound}

The ground state energy of $H_{N}$ is lower than the expectation value of $\tilde{H}_{N}$ in the ground state of $h_{N}(0)$, if we define $\tilde{H}_{N}$ to be the unitary equivalent of $H_{N}$ by the transformation 6):

$$
\begin{aligned}
& \tilde{H}_{N}=N^{2 / 3} \sum_{i=1}^{N} p_{i}^{2} / 2 m-N^{4 / 3} \sum_{i=1}^{N}\left|x_{i}\right|^{-1}+N^{1 / 3} \sum_{i<j}\left|x_{i}-x_{j}\right|^{-1} . \\
& \lim _{N \rightarrow \infty} N^{-7 / 3} \inf _{\psi,\|\psi\|=1}\left\langle\psi\left|H_{N}\right| \psi\right\rangle \leqq \lim _{N \rightarrow \infty} N^{-7 / 3}\left\langle\psi_{N}\left|\tilde{H}_{N}\right| \psi_{N}\right\rangle \\
& =(3 / 10 m)\left(3 \pi^{2}\right)^{2 / 3} \int \varrho_{\text {T.F. }}(x)^{2 / 3} d^{3} x-\int|x|^{-1} \varrho_{\text {T.F. }}(x) d^{3} x \\
& \quad+N^{-2} \lim _{N \rightarrow \infty}\left\langle\psi_{N}\left|\sum_{i<j}\right| x_{i}-\left.x_{j}\right|^{-1} \mid \psi_{N}\right\rangle .
\end{aligned}
$$

1 We could even take any $w \in L^{5 / 2}\left(\mathbb{R}^{3}\right)$, as has been shown by W. Thirring [5]. 
[That the kinetic energy converges to the corresponding T.F.-term follows by using the concavity of the ground state energy in $1 / \mathrm{m}$ and the same arguments which lead to (19).]

$\psi_{N}\left(x_{1} \ldots x_{N} ; s_{1} \ldots s_{N}\right)$, the ground state of $h_{N}(0)$, is a determinant of one-particlewavefunctions $\varphi_{N, i}\left(x_{j}, s_{j}\right)$. In the evaluation of an upper bound to the electron repulsion we may omit the exchange-terms (which are negative), and add the positive "self energies" of the $\varphi_{N, i}$ 's:

$$
\frac{1}{2} \sum_{s, s^{\prime}} \int\left|\varphi_{N, i}(x, s)\right|^{2}\left|x-x^{\prime}\right|^{-1}\left|\varphi_{N, i}\left(x^{\prime}, s^{\prime}\right)\right|^{2} d^{3} x d^{3} x^{\prime}
$$

We obtain

$$
N^{-2}\left\langle\psi_{N}\left|\sum_{i<j}\right| x_{i}-\left.x_{j}\right|^{-1} \mid \psi_{N}\right\rangle \leqq \frac{1}{2} \int \varrho_{N}(x)|x-y|^{-1} \varrho_{N}(y) d^{3} x d^{3} y .
$$

We decompose $|x-y|^{-1}$ into three parts:

$$
|x-y|^{-1}=V_{s}(x-y)+V_{r}(x, y)+V_{l}(x, y),
$$

where

$$
\begin{aligned}
& V_{s}(x-y)=\left\{\begin{array}{lll}
|x-y|^{-1}-\varepsilon^{-1} & \text { for } & (x, y) \in A_{\varepsilon}=\{(x, y):|x-y|<\varepsilon\} \\
0 & & (x, y) \notin A_{\varepsilon}
\end{array}\right. \\
& V_{r}(x, y)= \begin{cases}\varepsilon^{-1} & (x, y) \in A_{\varepsilon} \\
0 & (x, y) \in A_{R}=\{(x, y):|x|>R,|y|>R\} \\
|x-y|^{-1} & (x, y) \notin A_{\varepsilon} \cup A_{R}\end{cases} \\
& V_{l}(x, y)= \begin{cases}|x-y|^{-1} & (x, y) \in A_{R} \\
0 & (x, y) \notin A_{R} .\end{cases}
\end{aligned}
$$

The interaction due to the singular part is bounded because of Young's inequality [8]:

$$
\int \varrho_{N}(x) V_{s}(x-y) \varrho_{N}(y) \leqq\left\|V_{s}\right\|_{5 / 4}\left\|\varrho_{N}\right\|_{5 / 3}^{2},
$$

$\left\|\varrho_{N}\right\|_{5 / 3}$ is bounded by the kinetic energy [9]:

$$
\left\|\varrho_{N}\right\|_{5 / 3} \leqq(5 / 3)^{3 / 5}(4 / 3 \pi)^{2 / 5} N^{-5 / 3} \sum_{i=1}^{N}\left\langle\varphi_{N, i}\left|p^{2}\right| \varphi_{N, i}\right\rangle,
$$

which converges and is therefore bounded.

$V_{r}(x, y)$ is uniformly approximable by finite sums of terms $\chi_{\alpha}(x) \chi_{\beta}(y)$ and there the integrals converge [following (19)]:

$$
\lim _{N \rightarrow \infty} \int \varrho_{N}(x) V_{r}(x, y) \varrho_{N}(y) d^{3} x d^{3} y=\int \varrho_{\text {T.F. }}(x) V_{r}(x, y) \varrho_{\text {T.F. }}(y) d^{3} x d^{3} y .
$$

$V_{l}(x, y)$ is the mutual interaction of electrons which are far away from the nucleus:

$$
\int \varrho_{N}(x) V_{l}(x, y) \varrho_{N}(y) d^{3} x d^{3} y \leqq \sup _{x, y}\left|V_{l}(x, y)\right|\left(\int_{|x|>R} \varrho_{N}(x) d^{3} x\right)^{2} .
$$


From $\lim _{N \rightarrow \infty} \int_{|x|<R} \varrho_{N}(x) d^{3} x=\int_{|x|<R} \varrho_{\text {T.F. }}(x) d^{3} x$ and $\left\|\varrho_{N}\right\|_{1}=1$, it follows that $\int_{|x|>R} \varrho_{N}(x) d^{3} x$ converges to $\int_{|x|>R}^{|x|<R} \varrho_{\text {T.F. }}(x) d^{3} x$ and is therefore bounded by any constant close to zero, if $R$ is large enough.

By taking the limits $\varepsilon \rightarrow 0$ and $R \rightarrow \infty$ after $N \rightarrow \infty$, we conclude:

$$
\lim _{N \rightarrow \infty} \varrho_{N}(x)|x-y|^{-1} \varrho_{N}(y) d^{3} x d^{3} y=\int \varrho_{\text {T.F. }}(x)|x-y|^{-1} \varrho_{\text {T.F. }}(y) d^{3} y,
$$

and that establishes the convergence of the upper bound, which equals the lower bound:

$$
\lim _{N \rightarrow \infty} N^{-7 / 3} \inf _{\psi,\|\psi\|=1}\left\langle\psi\left|H_{N}\right| \psi\right\rangle=E_{\text {T.F. }} .
$$

Acknowledgements. This work was part of a doctoral thesis at the Institut für theoretische Physik der Universität Wien. I am indebted to Prof. W. Thirring for pointing out the problems and for the supervision of my work.

\section{References}

1. Lieb,E.H., Simon, B.: Phys. Rev. Letters 31, 681-683 (1973)

2. Hertel, P., Lieb, E.H., Thirring, W.: J. Chem. Phys. 62, 3355-3356 (1975)

3. Baumgartner, B.: Bound States in the Strong-Coupling-Limit. To appear in Acta Phys. Austriaca

4. Gombas, P.: Die statistische Theorie des Atoms und ihre Anwendungen. Wien: Springer 1949

5. Thirring, W.: Vorlesungen über mathematische Physik T8: Quantenmechanik großer Systeme. Lecture Notes

6. Griffiths, R. B.: J. Math. Phys. 5, 1215-1222 (1964)

7. Hepp, K., Lieb,E.H.: Phys. Rev. A8, 2517-2525 (1973)

8. Brascamp, H.J., Lieb, E. H., Luttinger, J.: J. Funct. Anal. 17, 227 (1974)

9. Lieb, E. H., Thirring, W.: A Bound for the Kinetic Energy of Fermions which Proves the Stability of Matter. Phys. Rev. Letters 35, 187-189 (1975)

Communicated by W. Hunziker

Received November 10, 1975 
